\title{
Privaciones múltiples en la Argentina: diferencias entre hogares con jefatura masculina y hogares con jefatura femenina ${ }^{1}$
}

\author{
Carla Arévalo² \\ Jorge A. Paz ${ }^{3}$
}

Recibido: 30/06/2016

Aceptado: 08/09/2016

\section{Resumen}

En este trabajo se examinan las diferencias de género en la probabilidad de sufrir privaciones múltiples. Para medir la pobreza en la Argentina se aplica la metodología multidimensional propuesta por Alkire y Foster (2007 y 2011) a datos de la Encuesta Permanente de Hogares (EPH). La estrategia empírica usada para identificar los determinantes profundos de la pobreza y de la brecha de género incluye la descomposición del índice de pobreza multidimensional $\left(M_{0}\right)$ y descomposiciones microeconométricas basadas en Blinder (1973), Oaxaca (1973) y Yun (2005).

Se encuentra que efectivamente existe una brecha de privaciones entre los hogares con jefatura masculina y los hogares con jefatura femenina y que esta no solo no

\footnotetext{
Los autores agradecen a los participantes del Seminario Pobreza en la Argentina, realizado el 22 de diciembre de 2015, especialmente a Emma Santos. También agradecen las observaciones realizadas por dos árbitros a la presente versión del trabajo. Los errores y las omisiones que pudiera haber en este documento son de exclusiva responsabilidad de los autores.

2 Consejo Nacional de Investigaciones Científicas y Técnicas (CONICET), Instituto de Estudios Laborales y del Desarrollo Económico (IELDE), Facultad de Ciencias Económicas, Universidad Nacional de Salta, Argentina. Correo electrónico: c.arevalo@conicet.gov.ar.

3 Consejo Nacional de Investigaciones Científicas y Técnicas (CONICET), Instituto de Estudios Laborales y del Desarrollo Económico (IELDE), Facultad de Ciencias Económicas, Universidad Nacional de Salta, Argentina. Correo electrónico: jpaz@conicet.gov.ar.
} 
disminuyó sino que aumentó en la última década. Se observa además que los hogares encabezados por mujeres no son más proclives a la pobreza por una actitud discriminatoria hacia ellas, sino porque las jefas de hogar tienen ciertas desventajas desde el punto de vista de las dotaciones básicas.

Palabras clave: pobreza, privaciones, pobreza multidimensional, género, desigualdad.

\begin{abstract}
This work examines gender differences in the probability of suffering multiple deprivations. To measure poverty in Argentina, the multidimensional methodology proposed by Alkire and Foster (2007 and 2011) is applied to data from the Permanent Household Survey. The empirical strategy used to identify the underlying determinants of poverty and the gender gap include the decomposition of the multidimensional poverty index $\left(M_{0}\right)$ and microeconometric decompositions on the basis of Blinder (1973), Oaxaca (1973) and Yun (2005)

It was found that there is indeed a gap in deprivations between male-headed and female-headed households and that, rather than narrowing, this gap has in fact widened over the past decade. It was also observed that female-headed households are not more susceptible to poverty by reason of discrimination, but rather because women who are heads of household suffer certain disadvantages in terms of basic skills endowment.
\end{abstract}

Keywords: poverty, privation, multidimensional poverty, gender, inequality.

\title{
Résumé
}

Dans cette étude, l'auteure analyse des différences entre les sexes en termes de probabilité de subir des privations multiples. Pour mesurer la pauvreté en Argentine, la méthodologie multidimensionnelle proposée par Alkire et Foster (2007 et 2011) a été appliquée aux données de l'enquête permanente sur les ménages. La stratégie empirique utilisée pour cerner les déterminants profonds de la pauvreté et de l'écart entre les sexes inclut la décomposition de l'indice de pauvreté multidimensionnelle $\left(M_{0}\right)$, ainsi que des décompositions micro économétriques basées sur Blinder (1973), Oaxaca (1973) et Yun (2005).

Le constat est qu'il existe effectivement un écart en termes de privations entre les ménages dirigés par des hommes et les ménages dirigés par des femmes, et que cet écart non seulement ne s'est pas réduit, mais aussi qu'il s'est creusé durant la dernière décennie. On observe également que les ménages dirigés par des femmes ne montrent pas une plus grande tendance à la pauvreté en raison d'une attitude de discrimination à leur égard, mais parce que les femmes-chefs de ménage sont confrontées à un certain nombre de désavantages du point de vue des ressources élémentaires.

Mots clés: pauvreté, privations, pauvreté multidimensionnelle, genre, inégalité 


\section{Introducción}

En la Cumbre de septiembre de 2015, los Estados Miembros de las Naciones Unidas aprobaron la Agenda 2030 para el Desarrollo Sostenible, que incluye un conjunto de 17 Objetivos de Desarrollo Sostenible (ODS) para poner fin a la pobreza, luchar contra la desigualdad y la injusticia y hacer frente al cambio climático. El primer ODS, "poner fin a la pobreza en todas sus formas en todo el mundo", alude de manera directa a las múltiples formas que asume la pobreza. Aunque a lo largo de este trabajo se hace referencia a varios ODS, su motivación básica es contribuir al logro del primer objetivo y también a una parte del quinto, que consiste en "lograr la igualdad de género y empoderar a todas las mujeres y las niñas".

En un estudio previo (Arévalo y Paz, 2015), se encontró que en la Argentina, a principios del año 2015, la brecha de privaciones múltiples entre hogares con jefatura masculina y hogares con jefatura femenina era amplia a nivel descriptivo y condicionalmente significativa, es decir, aún después de controlar los factores que determinan la pobreza multidimensional. Estudios similares sobre otros países revelan una situación análoga: diferencias de pobreza según el género de los jefes de hogar, desfavorable para las mujeres en todos los casos (Agbodji, Batana y Ouedraogo, 2013), y desventaja similar a la indicada por las medidas convencionales de pobreza basadas en el ingreso o en el consumo (Rogan, 2014).

No obstante la importancia de esos hallazgos, no se ha encontrado en la literatura un análisis de los factores subyacentes a las privaciones propiamente dichas y mucho menos una descomposición de dichos factores para detectar el origen de la diferencia. Más precisamente, no se han encontrado estudios que permitan establecer si las brechas se deben a diferencias en las características de los hogares encabezados por personas de uno u otro sexo o a prácticas discriminatorias que afectan en mayor medida a los hogares dirigidos por mujeres. Las políticas contra la pobreza podrían mejorarse considerablemente. Ese es uno de los propósitos de este artículo.

El objetivo principal de este trabajo es cuantificar la magnitud de la brecha de privaciones múltiples en hogares con jefatura masculina y femenina en la Argentina y determinar si la disparidad que se detecta a nivel descriptivo y condicional obedece a factores que podrían denominarse genéricamente "dotaciones" (diferencias relativas al nivel educativo, la edad o el estado civil del jefe, la ubicación del hogar o la carga demográfica) o a prácticas discriminatorias, entendiéndose por tales aquellas que suponen un tratamiento desigual ante dotaciones similares.

El artículo se divide en cinco secciones, incluida esta introducción. En la segunda se revisa la literatura relacionada con los objetivos que se persiguen, mientras que en la tercera se describe la fuente de los datos y se desarrolla la metodología usada para su tratamiento. En la cuarta sección se presentan y discuten los resultados del estudio. La quinta y última contiene las principales conclusiones de la investigación y algunas preguntas que se derivan de sus principales hallazgos. 


\section{A. Revisión de la literatura}

El presente documento se inscribe en el grupo de trabajos basados en el aporte seminal de Alkire y Foster (2011). Desde ese momento y hasta la fecha, se han publicado diversos estudios empíricos relacionados con el tema, no solo en el ámbito académico, sino también en el de oficinas de los estados nacionales interesadas en conocer la situación de bienestar de la población de sus países a través de medidas sintéticas o de resumen. Battiston y otros (2009) realizaron un trabajo pionero sobre el tema en América Latina.

Sin embargo, el interés en la medición de la pobreza con un enfoque que tuviera en cuenta otras variables además del ingreso se remonta a mediados de la década de 1970 (por ejemplo Hicks y Streeten, 1979). Los autores latinoamericanos Beccaria y Minujin (s/f), Boltvinik (1992) y Cid (1994), entre otros, comenzaron a aplicar esas ideas al corpus metodológico llamado "medición integrada de la pobreza". En esos estudios se consideraba pobres a los hogares y las personas que presentaban necesidades básicas insatisfechas (NBI), ingresos insuficientes para cubrir una canasta de bienes y servicios (enfoque de la línea de pobreza), o ambas privaciones simultáneamente (Feres y Mancero, 2001)4. Esa visión, defendida y refrendada por Ravallion (1996 y 2011), está en la base de los ODS.

Los llamados índices compuestos, como el índice de pobreza de capacidad (IPC) y el índice de pobreza humana (IPH) (PNUD, 1996 y 1997), fueron los intentos que siguieron a esa serie de estudios y constituyen los antecedentes de las medidas usadas en este trabajo. Concretamente, este documento se basa en el enfoque axiomático (Sen, 1976) para la medición de la pobreza multidimensional, que reconoce los aportes pioneros de Chakravarty (1997), Foster y Sen (1997), Foster (1998), Tsui (2002), Bourguignon y Chakravarty (2003), Chakravarty y D'Ambrosio (2006), Alkire y Foster (2007, 2011), Bossert, Chakravarty y D'Ambrosio (2009) y Maasoumi y Lugo (2008).

En la Argentina, los antecedentes de cómputos de pobreza realizados con este enfoque no son muchos: Conconi y Ham (2007) , Conconi (2009), Tuñón y González (2013), López y Safojan (2013), Paz (2014) y Arévalo y Paz (2015). Desde el punto de vista del interés temático, el primero de estos es el que más se aproxima a lo que se pretende hacer en este documento: analizar las privaciones múltiples desde una perspectiva de género. Por otra parte, Tuñón y González (2013) estiman la incidencia y la composición de la pobreza infantil mediante el denominado enfoque de los derechos, usando la propuesta de UNICEF-CEPAL (CEPAL, 2010 y 2013) y datos de la Encuesta sobre la Deuda Social de la Argentina (EDSA).

4 Boltvinik (1992) agrega la pobreza de tiempo, adelantándose casi 20 años a las primeras investigaciones iniciadas por el Levy Economics Institute en el mundo. Véase Levy Economics Institute of Bard College [en línea] http://www. levyinstitute.org/topics/time-poverty.

5 En realidad, el estudio de Conconi y Ham (2007) estaría entre los trabajos que usan los métodos estadísticos multivariantes (componentes principales, conglomerados y análisis factorial, entre otros) para reducir las dimensiones. El aporte de estos autores consiste en utilizar el análisis factorial para identificar "funcionamientos" (functioning). 
Las contribuciones más recientes sobre el tema pueden hallarse en los trabajos de Santos y otros (2015) sobre América Latina y el Caribe y en los estudios de Paz (2014), Arévalo y Paz (2015) y Salvia, Bonfiglio y Vera (2015) con respecto a la Argentina. En el primero de esos estudios se propone un nuevo Índice de Pobreza Multidimensional para América Latina, que se calcula para 17 países de la región en dos puntos en el tiempo: alrededor de 2005 y de 2012. Los autores encontraron que alrededor del $28 \%$ de las personas eran multidimensionalmente pobres en 2012, aunque con una gran variabilidad entre países, y que la pobreza se redujo en la mayoría de las naciones en el período analizado, tanto en términos de incidencia como de intensidad. Sin embargo, se mantuvo la disparidad entre zonas rurales y urbanas.

Paz (2014) presenta una primera medición de la pobreza multidimensional en la Argentina, que abarca grandes ciudades y centros urbanos de menor envergadura demográfica. Los resultados dan cuenta de una fuerte asimetría entre las ciudades argentinas, con tasas de pobreza multidimensional que van de un máximo del 40,3\% a un mínimo del 2,5\% de la población. Arévalo y Paz (2015) analizan la evolución de la pobreza en la Argentina en la última década (2005-2015) y encuentran que esta disminuyó durante el período examinado y que la disminución fue más intensa en el primer quinquenio.

En todos los casos resulta difícil comparar los resultados obtenidos en esos estudios y las estimaciones realizadas para el presente, debido a que las medidas de pobreza multidimensional son muy sensibles a las decisiones metodológicas adoptadas. Así, por ejemplo, un simple cambio en la cantidad y el tipo de dimensiones, privaciones e indicadores, arroja resultados diferentes y los valores obtenidos pierden comparabilidad.

Los estudios de privaciones múltiples con foco en el género son menos numerosos que los que podrían denominarse "temáticos", como por ejemplo los que se ocupan de los pueblos indígenas o de los niños. No obstante, los hallazgos de los autores que se ocuparon de género son sugestivos. Agbodji, Batana y Ouedraogo (2013) desarrollan un indicador de diferencias de género para identificar cuál o cuáles de las dimensiones de la pobreza analizadas contribuyen en mayor medida a la creación de la brecha de género. Rogan (2014) destaca la persistencia de la brecha entre los hogares encabezados por personas de distinto sexo en un contexto de reducción generalizada de los niveles de pobreza en Sudáfrica. Encuentra que la pobreza multidimensional de las personas que viven en hogares encabezados por mujeres es mucho más elevada que la del resto de la población e incluso alcanza el $100 \%$ en algunos casos.

En el estudio de Mahoozi (2015) se explica que, en 2008 en la República Islámica del Irán, la población que habitaba en hogares con jefatura femenina y residía en zonas rurales estaba en amplia desventaja comparada con aquella que lo hacía en hogares con jefatura masculina y en zonas urbanas. La pobreza en los hogares con jefatura femenina duplicaba con creces la pobreza en los hogares con jefatura masculina.

Entre los estudios sobre pobreza multidimensional en países latinoamericanos que incorporan un análisis de género se destacan los trabajos de Barros, Carvalho y Franco (2006), Cortés (1997) y Mideros (2012). De acuerdo con el primero, en el Brasil en el año 2003, la 
proporción de hogares encabezados por mujeres entre los hogares más pobres era mucho más alta que en el promedio de la población en su conjunto. Además, la incidencia de la pobreza multidimensional en las familias encabezadas por mujeres era mayor que la media nacional (del $28 \%$ y el $25 \%$, respectivamente).

Cortés (1997) analiza la relación entre género y pobreza por medio de dos indicadores: la proporción de hogares pobres según el sexo del jefe y la incidencia de la pobreza en hogares con perceptores de ingresos monetarios exclusivamente femeninos en comparación con aquellos donde hay de ambos sexos o solo hombres. El análisis se basa en datos de México relativos al año 1992 y en el método integrado de medición de la pobreza. Se encuentra que esta es menor cuando la presencia femenina cobra relevancia: ambos indicadores muestran que la pobreza es menor en los hogares en que la mujer administra los recursos. Según el autor, esos resultados concuerdan con el argumento de la eficiencia de la mujer en la administración del hogar.

Por último, en un estudio sobre el Ecuador, Mideros (2012) encuentra que la probabilidad de ser multidimensionalmente pobre es mayor para las mujeres que para los hombres, así como para las personas que residen en hogares con jefatura femenina, en comparación con las personas que residen en hogares con jefatura masculina. Aunque las diferencias halladas por este autor son bajas, todas resultan estadísticamente significativas.

\section{B. Datos y metodología}

\section{Datos, dimensiones e indicadores}

Se utilizan datos de los años 2005 y 2015 de la Encuesta Permanente de Hogares (EPH). La EPH es un programa nacional de producción sistemática y permanente de indicadores sociales que lleva a cabo el Instituto Nacional de Estadística y Censos (INDEC) con la colaboración de las direcciones de estadística de las provincias de la Argentina. La información recolectada permite conocer las características sociodemográficas y socioeconómicas de la población. La encuesta se aplica desde 1973, dos veces al año (mayo y octubre) en su modalidad puntual (1973-2003) y de manera continua desde 2003 hasta la fecha. Se han llegado a cubrir 31 aglomerados urbanos y un área urbano-rural. La representatividad aproximada de la EPH es del 70\% de la población total de la República Argentina.

Para analizar las privaciones múltiples se seleccionaron cinco dimensiones definidas sobre la base del enfoque de las capacidades (capabilities) y teniendo en cuenta la agenda que determina los ODS para los países. Como se puede observar en el cuadro 1, las dimensiones seleccionadas son: i) capacidad económica, ii) vivienda adecuada, iii) saneamiento básico, iv) inclusión-integración social, y v) acceso a la salud. Cabe aclarar que las dimensiones tienen aquí un objetivo instrumental, en la medida que constituyen una orientación conveniente para la selección de indicadores. Ese es el criterio aplicado a lo largo del presente estudio. 


\section{Cuadro 1}

\section{Origen de los indicadores seleccionados para medir la pobreza multidimensional en la Argentina}

\begin{tabular}{|c|c|c|c|c|}
\hline Capacidad & Dimensión & Privación & Indicadores & ODS/meta \\
\hline $\begin{array}{l}\text { Ingresos } \\
\text { adecuados } \\
\text { actuales }\end{array}$ & $\begin{array}{l}\text { Capacidad } \\
\text { económica }\end{array}$ & $\begin{array}{l}\text { Pobre } \\
\text { monetario }\end{array}$ & $\begin{array}{l}\text { Ingresos familiares } \\
\text { por debajo del } \\
\text { umbral (canasta } \\
\text { básica total) }\end{array}$ & $\begin{array}{l}\text { ODS 1-Poner fin a la pobreza en todas } \\
\text { sus formas y en todo el mundo }\end{array}$ \\
\hline $\begin{array}{l}\text { Capacidad } \\
\text { de procurarse } \\
\text { un ingreso }\end{array}$ & & $\begin{array}{l}\text { Bajo nivel } \\
\text { educativo del } \\
\text { jefe del hogar }\end{array}$ & $\begin{array}{l}\text { Menos de siete } \\
\text { años de escolaridad } \\
\text { formal }\end{array}$ & $\begin{array}{l}\text { ODS 2-Poner fin al hambre, lograr } \\
\text { la seguridad alimentaria y la mejora } \\
\text { de la nutrición y promover } \\
\text { la agricultura sostenible }\end{array}$ \\
\hline $\begin{array}{l}\text { Contar con } \\
\text { un ingreso } \\
\text { estable } \\
\text { y seguro }\end{array}$ & & $\begin{array}{l}\text { Estrategias } \\
\text { precarias }\end{array}$ & $\begin{array}{l}\text { Subsidio o ayuda } \\
\text { social de cualquier } \\
\text { fuente (gobierno, } \\
\text { iglesia, entre otros) o } \\
\text { caridad de personas } \\
\text { o instituciones }\end{array}$ & $\begin{array}{l}\text { Meta 11.1-De aquí a 2030, asegurar } \\
\text { el acceso de todas las personas } \\
\text { a viviendas y servicios básicos } \\
\text { adecuados, seguros y asequibles } \\
\text { y mejorar los barrios marginales }\end{array}$ \\
\hline \multirow{3}{*}{$\begin{array}{l}\text { Vivir en una } \\
\text { vivienda } \\
\text { adecuada }\end{array}$} & \multirow{3}{*}{$\begin{array}{l}\text { Vivienda } \\
\text { adecuada }\end{array}$} & \multirow{3}{*}{$\begin{array}{l}\text { Vivienda } \\
\text { inadecuada I }\end{array}$} & Hacinamiento crítico & \\
\hline & & & $\begin{array}{l}\text { No tiene cuarto } \\
\text { de cocina }\end{array}$ & \\
\hline & & & Zona de basural & \\
\hline \multirow{2}{*}{$\begin{array}{l}\text { Tener acceso } \\
\text { al saneamiento } \\
\text { básico }\end{array}$} & \multirow{2}{*}{$\begin{array}{l}\text { Saneamiento } \\
\text { básico }\end{array}$} & \multirow{2}{*}{$\begin{array}{l}\text { Vivienda } \\
\text { inadecuada II }\end{array}$} & Baño sin desagüe & \multirow{2}{*}{$\begin{array}{l}\text { ODS 6-Garantizar la disponibilidad } \\
\text { - y la gestión sostenible del agua } \\
\text { y el saneamiento para todos }\end{array}$} \\
\hline & & & $\begin{array}{l}\text { Sin agua } \\
\text { en la vivienda }\end{array}$ & \\
\hline \multirow[t]{5}{*}{$\begin{array}{l}\text { Estar } \\
\text { socialmente } \\
\text { incluido }\end{array}$} & \multirow[t]{5}{*}{$\begin{array}{l}\text { Inclusión } \\
\text { económica } \\
\text { y social }\end{array}$} & $\begin{array}{l}\text { Niños, niñas } \\
\text { y adolescentes } \\
\text { (5-17 años) } \\
\text { excluidos }\end{array}$ & $\begin{array}{l}\text { Miembro del hogar } \\
\text { entre } 5 \text { y } 17 \text { años de } \\
\text { edad que no asiste a } \\
\text { la escuela }\end{array}$ & $\begin{array}{l}\text { ODS 4-Garantizar una educación } \\
\text { inclusiva y equitativa de calidad } \\
\text { y promover oportunidades de } \\
\text { aprendizaje permanente para todos }\end{array}$ \\
\hline & & $\begin{array}{l}\text { Jóvenes } \\
\text { (18-24 años) } \\
\text { excluidos }\end{array}$ & $\begin{array}{l}\text { Joven (18-24 años) } \\
\text { que no estudia, no } \\
\text { trabaja, ni busca } \\
\text { trabajo }\end{array}$ & $\begin{array}{l}\text { ODS 8-Promover el crecimiento } \\
\text { económico sostenido, inclusivo y } \\
\text { sostenible, el empleo pleno y productivo } \\
\text { y el trabajo decente para todos }\end{array}$ \\
\hline & & & & $\begin{array}{l}\text { Meta 1.3-Implementar a nivel nacional } \\
\text { sistemas y medidas apropiados de } \\
\text { protección social para todos, incluidos } \\
\text { niveles mínimos, y, de aquí a 2030, } \\
\text { lograr una amplia cobertura de las } \\
\text { personas pobres y vulnerables }\end{array}$ \\
\hline & & $\begin{array}{l}\text { Adultos } \\
\text { (25-64 años) } \\
\text { excluidos }\end{array}$ & Desocupado & \multirow[t]{2}{*}{$\begin{array}{l}\text { Meta 10.4-Adoptar políticas, } \\
\text { especialmente fiscales, salariales } \\
\text { y de protección social, y lograr } \\
\text { progresivamente una mayor igualdad }\end{array}$} \\
\hline & & $\begin{array}{l}\text { Adultos } \\
\text { mayores (65 } \\
\text { años o más) } \\
\text { excluidos }\end{array}$ & $\begin{array}{l}\text { Adultos mayores } \\
\text { (65 años o más) sin } \\
\text { cobertura previsional }\end{array}$ & \\
\hline $\begin{array}{l}\text { Gozar de } \\
\text { buena salud }\end{array}$ & Salud & & $\begin{array}{l}\text { Sin cobertura } \\
\text { médica }\end{array}$ & $\begin{array}{l}\text { ODS 3-Garantizar una vida sana } \\
\text { y promover el bienestar de todos } \\
\text { a todas las edades }\end{array}$ \\
\hline
\end{tabular}

Fuente: Elaboración propia, sobre la base de J. A. Paz, "Pobreza multidimensional en la Argentina. Asimetrías regionales (parte I)", Documentos de Trabajo, No 11, Salta, Instituto de Estudios Laborales y del Desarrollo Económico (IELDE), 2014. 
La dimensión "capacidad económica" incluye tres privaciones: i) ingresos insuficientes, ii) (in)capacidad del jefe del hogar de generar ingresos, iii) (in)capacidad del grupo familiar de mantener un ingreso estable y más o menos seguro, de ser autosuficiente o ambos. Cada privación se asocia con un indicador: ingresos familiares por debajo del mínimo indispensable para cubrir los gastos correspondientes a la canasta básica total (que equivale a la pobreza monetaria, por ingresos o por consumo), nivel educativo del jefe del hogar por debajo de los siete años y estrategias hogareñas precarias conforme la definición del concepto de Cid (2013a y 2013b). En forma resumida puede decirse que las "estrategias precarias" son aquellas que denotan una situación de debilidad o carencia. Todos los indicadores utilizados tienen una connotación negativa y son útiles para controlar el desempeño del país en términos de los ODS 1 y 2: "poner fin a la pobreza y al hambre".

En la dimensión "vivienda adecuada" se incorporaron tres privaciones, cada una con su correspondiente indicador:i) hacinamiento crítico, según el concepto de las oficinas estadísticas de la Argentina ${ }^{6}$, ii) vivienda sin cuarto de cocina y iii) emplazamiento de la vivienda cerca de una zona de basurales. Esta dimensión es útil para monitorear específicamente la meta 1 del ODS 11, que solicita a los países que trabajen para lograr el acceso de todas las personas a viviendas y servicios básicos adecuados y mejorar los barrios marginales.

Para dar contenido a la dimensión "inclusión económica y social", se utilizó el concepto de "frontera de inclusión". Esta supone que una persona está incluida económica y socialmente (o tiene mayores probabilidades de estarlo) si participa en instituciones sociales relacionadas de manera directa con la etapa del ciclo vital en la que se encuentra: los niños en la escuela, los jóvenes en un establecimiento educativo o en el mercado laboral, los adultos en el mercado laboral y los adultos mayores en la seguridad social.

Con respecto a los hogares que no tienen miembros de alguno de los grupos de edad y en los que, por ende, se desconoce cuál hubiese sido la situación en caso de tenerlos, se resolvió suponer que no están privados en el indicador referido a ese grupo particular.

Por último, para captar la dimensión "acceso a la salud", se tomó como indicador la cobertura de obra social o medicina prepaga. Se debe aclarar en este caso que el sistema de salud pública de la Argentina permite el acceso a todos los individuos, independientemente de su nivel de cobertura. No obstante, la falta de protección en este aspecto, fuertemente ligada a una inserción precaria en el mercado de trabajo, conlleva una presión mayor sobre el sistema de salud pública.

\section{Medidas sintéticas}

La unidad de identificación, entendida como la unidad que se identifica como multidimensionalmente pobre, es el hogar. Por lo tanto, todos sus miembros comparten la condición de privado o pobre. Algunos indicadores son atributos naturales del hogar,

6 Se entiende por "hacinamiento crítico" la situación en la cual hay más de tres personas por cuarto destinado a dormitorio en la vivienda. Véase Instituto Nacional de Estadística y Censos (INDEC) [en línea] http://www.indec. mecon.ar/glosario.asp. 
pero otros aluden a la situación de un integrante en particular. Por ejemplo, según el diseño del indicador, estar desempleado es un estado exclusivo de los adultos que tienen entre 25 y 64 años de edad. Debido a la unidad de identificación elegida, si en el hogar existe un desempleado (entre 25 y 64 años) todos los miembros de ese hogar resultarán privados en ese indicador. Sin embargo, los resultados se reportan y analizan a nivel individual o, en otras palabras, la unidad de análisis son los individuos.

Tras definir la unidad de identificación se debe, en primer lugar, establecer líneas de corte para cada indicador, cada una de las cuales constituye una condición de privación y establece la categoría de privado o no privado de la unidad. Luego se determina un número de privaciones, de manera que aquellos hogares que alcancen o superen ese nivel se considerarán pobres por privaciones múltiples.

Suelen mencionarse dos enfoques para la fijación del umbral multidimensional, conocido en la literatura como factor : i) el primero, llamado de la "unión", establece que un hogar es pobre si presenta al menos una de las privaciones consideradas, ii) el segundo, denominado "intersección", exige que un hogar tenga todas las privaciones para ser considerado pobre. Como es sencillo imaginar, el enfoque de la unión sobrestimará el nivel de pobreza, mientras que el de la intersección lo subestimará. Entre estos dos criterios se encuentra uno intermedio, que es el que se utiliza aquí. Para ello se incluyen 13 indicadores (véase el cuadro 1) y se selecciona $k=3$. Es decir que será considerado pobre el individuo que tenga tres o más privaciones, o bien, un $23 \%$ del total de privaciones.

Una de las propiedades deseables de las medidas de pobreza multidimensional es la monotonicidad dimensional, que supone que el índice sintético aumente cuando a una persona ya clasificada como pobre se le agregue una privación adicional. Debido a que la tasa de recuento multidimensional, reconocida como $H$ en la literatura, no cumple con esta propiedad, se incorpora al análisis la tasa de recuento multidimensional ajustada, un indicador que se construye multiplicando el valor de $H$ la proporción de pobres, por " $A$ ", que simboliza el promedio de privaciones en la población pobre en proporción al total de privaciones consideradas. Este indicador, conocido como $M_{0}$, puede interpretarse como la cantidad total de privaciones sufridas por los pobres dividida por la cantidad máxima de privaciones que la totalidad de las personas podrían padecer (Alkire y Foster, 2007).

La medida $M_{0}$ tiene además otra propiedad interesante y muy útil: puede descomponerse en dimensiones y subgrupos para detectar la prevalencia de privaciones o de subgrupos más afectados entre los pobres. Un aspecto relevante de la medición de la pobreza multidimensional es la asignación normativa de un peso o valor a cada privación. Atkinson (2003) sugiere usar ponderaciones similares para cada dimensión dado que ello facilita la interpretación de los resultados. De acuerdo con esa recomendación se seleccionaron ponderadores iguales para todos los indicadores, otorgando igual grado de relevancia a cada una de las privaciones identificadas aquí.

Para evaluar la posibilidad de relaciones de sustitución y de complementariedad entre los indicadores se computaron las correlaciones de Spearman entre ellos. Un coeficiente negativo implica que los indicadores pueden sustituirse y uno positivo denota 
complementariedad (Gordon y otros, 2003). En este trabajo se observó que ninguna relación de sustitución supera el 2,6\% y, aunque son estadísticamente significativas, resultan despreciables. Así, se consideró que todas las privaciones tienen igual importancia y su provisión genera la misma ganancia de bienestar.

Obviamente, con los ponderadores elegidos, el peso de las dimensiones varía debido a las diferencias en la cantidad de indicadores por dimensión. Por ejemplo, la dimensión "inclusión social" pesa más que el resto $(31 \%)$ porque se considera relevante para una persona formar parte de las instituciones correspondientes según la etapa del ciclo de vida. La educación en la niñez y la juventud podría ser una herramienta importante en el presente de los niños y jóvenes pero lo será sobre todo en el futuro, para evitar caer en la vulnerabilidad y posiblemente en la pobreza. A su vez, el desempleo es uno de los factores de mayor impacto asociados a la pobreza, porque afecta profundamente el presente de los desempleados y de sus familias. Por último, los adultos mayores en condiciones de vulnerabilidad o pobreza que carecen de cobertura previsional difícilmente encontrarán los medios para mejorar su bienestar.

En este sentido, se sugiere que las mejoras en la inclusión social podrían tener repercusiones positivas en el resto de las dimensiones. Es por esto que se otorga mayor peso a la dimensión que conjuga estos aspectos con respecto a las de capacidad económica, vivienda adecuada y saneamiento básico y salud ( $23 \%$ cada una) 7 . En resumen, de las medidas multidimensionales proporcionadas en la literatura, en este documento se computan y reportan la tasa de incidencia $(H)$, la tasa de incidencia ajustada $\left(M_{0}\right)$, el promedio de privaciones como proporción del total por persona pobre $(A)$ y el promedio de privaciones absolutas por persona pobre $(I)$, con la convicción de que proporcionan una visión integral de la pobreza en la Argentina.

\section{Análisis condicional}

Para analizar los determinantes de las privaciones múltiples se aplica lo que en la literatura sobre pobreza multidimensional se denomina "regresión micro" (Alkire y otros, 2015). En el caso propuesto, la variable dependiente toma el valor uno si el hogar es multidimensionalmente pobre y cero en caso contrario.

Una vez estimados los parámetros de esa regresión micro con una regresión probit, se está en condiciones de descomponer esa brecha en la parte que estaría explicada por esos parámetros y la parte explicada por cada uno de los regresores. Esas variables caracterizan al hogar y la mayoría de ellas aluden a la situación del jefe: nivel educativo, edad, región de residencia, entre otras. El enfoque seleccionado para la descomposición es similar al de Blinder (1973), Oaxaca (1973) y Oaxaca y Ranson (1988) y fue desarrollado por Jann (2008). El método establece las proporciones de las brechas que se explican por cada uno de los componentes mencionados.

$7 \quad$ El saneamiento básico y la salud están íntimamente relacionados. En algunos casos, los indicadores de saneamiento incluso forman parte de la dimensión "salud", como por ejemplo en el Indicador de Pobreza Multidimensional construido por el Gobierno de Costa Rica. En el índice de ese país los indicadores "sin servicio de agua" y "sin eliminación de excretas" están incluidos en la dimensión "salud". Aunque en esta oportunidad se prefirió mantener ambas dimensiones separadas, ya que las políticas públicas tendientes a mejorar una y otra son diferentes, mantienen el mismo peso que la capacidad económica y la vivienda adecuada. 


\section{Resultados}

\section{Medidas sintéticas}

En el cuadro 2 se muestran las tres medidas sintéticas de privaciones múltiples para la Argentina. Como puede observarse, el porcentaje de personas multidimensionalmente pobres era del $25,2 \%$ en 2015 , es decir 10,7 puntos porcentuales menos que en 2005 (35,9\%). Se aprecia también que la situación es relativamente favorable para las personas que viven en hogares con jefatura masculina, en comparación con aquellas que residen en hogares encabezados por mujeres (34,3\% y $41 \%$, respectivamente). La brecha en la incidencia disminuyó de 6,7 a 5,5 puntos porcentuales entre 2005 y 2015.

Cuadro 2

Argentina: medidas sintéticas de pobreza multidimensional por género, 2005 y 2015

\begin{tabular}{|c|c|c|c|c|c|c|c|c|c|}
\hline \multirow{2}{*}{$\begin{array}{l}\text { Género } \\
\text { del jefe } \\
\text { de hogar }\end{array}$} & \multicolumn{3}{|c|}{$\begin{array}{l}\text { Tasa de incidencia } \\
\text { ajustada }\left(M_{0}\right)\end{array}$} & \multicolumn{3}{|c|}{$\begin{array}{c}\text { Tasa de } \\
\text { incidencia }(\mathrm{H})\end{array}$} & \multicolumn{3}{|c|}{$\begin{array}{c}\text { Promedio de privaciones } \\
\text { absolutas por persona pobre (I) }\end{array}$} \\
\hline & 2005 & 2015 & Cambio & 2005 & 2015 & Cambio & 2005 & 2015 & Cambio \\
\hline 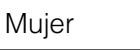 & 143 & 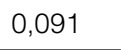 & & 4 & 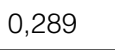 & & 4,5 & 4, &, \\
\hline 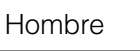 & 115 & 0,07 & 0 & & 0 & & 4, & & \\
\hline Diferencia & $0,028^{\star * *}$ & $0,021^{\star \star *}$ & & $0,067^{\star \star *}$ & $0,055^{\star \star *}$ & & $0,182^{\star * *}$ & $0,19^{\star \star \star}$ & \\
\hline Total & 0,122 & 0,077 & $0,045^{\star * \star}$ & 0,359 & 0,252 & $0,107^{\star \star *}$ & 4,42 & 3,986 & $0,434^{\star \star \star}$ \\
\hline
\end{tabular}

Fuente: Elaboración propia.

Nota: Diferencias intertemporales y de género significativas al ${ }^{* *} 1 \%$, ${ }^{* *} 5 \%$ y * $10 \%$.

Las medidas comparadas fueron calculadas con $\mathrm{k}=3$.

La medida que sintetiza la intensidad y la incidencia $\left(\mathrm{M}_{\mathrm{o}}\right)$ indica una reducción mayor entre las personas que viven en hogares con jefatura femenina, por lo que la brecha de género es levemente menor al final del período analizado. Dado que los cambios intertemporales de la intensidad de la pobreza multidimensional (promedio de privaciones entre pobres) son bajos, el efecto que predomina en la reducción de la pobreza corresponde a la incidencia.

Lo mismo ocurre con la diferencia entre los subgrupos: como el promedio de privaciones de los pobres que viven en hogares con jefatura masculina y sus pares con jefatura femenina no dista mucho del nivel promedio total (aproximadamente cuatro privaciones por pobre), es evidente que la diferencia en la pobreza multidimensional ajustada entre estos grupos se explica por la diferencia en $H$. En otras palabras, más que en una mayor intensidad, la diferencia de género radica principalmente en una mayor incidencia. 


\section{Descomposición de la desigualdad de género}

La diferencia de $\mathrm{M}_{0}$ por género asciende a o,021 puntos en el año 2015 (véase el cuadro 2). Esto significa que la pobreza multidimensional ajustada de las personas en hogares con jefatura femenina es un 30\% mayor que la de aquellas en hogares encabezados por un hombre.

En el cuadro 3 se presenta la estructura de esa brecha de género según las privaciones que la componen en los años 2005 y 2015. Se observa que en 2015 existen diferencias de género en todas las privaciones analizadas y que la desventaja de las personas que residen en hogares con jefatura femenina es recurrente. Más del 50\% de la brecha está explicada por: i) falta de cobertura médica, ii) pobreza monetaria y iii) percepción de subsidio, en ese orden de importancia.

Cuadro 3

Contribución de cada privación a la diferencia de género, 2005 y 2015

(En porcentajes)

\begin{tabular}{lcc}
\hline Privación & 2005 & 2015 \\
\hline Falta de cobertura médica & 22,5 & 21,3 \\
\hline Pobreza monetaria & 17,2 & 16,4 \\
\hline Ayuda social & 12,0 & 16,3 \\
\hline Jefe del hogar no educado & 14,4 & 10,2 \\
\hline Zona de basural & 4,8 & 7,5 \\
\hline Niños y adolescentes que no asisten a un centro educativo & 5,1 & 6,7 \\
\hline Falta de agua corriente & 4,2 & 6,6 \\
\hline Baño sin desagüe & 7,2 & 4,8 \\
\hline Desocupación & 0,0 & 3,1 \\
\hline Hacinamiento & 5,3 & 2,9 \\
\hline Adultos mayores sin cobertura previsional & 2,0 & 2,1 \\
\hline Falta de cuarto de cocina & 5,8 & 1,8 \\
\hline No estudia ni trabaja (nini) & $-0,6$ & 0,2 \\
\hline Total & 100 & 100 \\
\hline
\end{tabular}

Fuente: Elaboración propia.

En los dos años analizados, la privación que más contribuye a la diferencia de género es la falta de cobertura médica. Esto no solo refleja la mayor desventaja de las personas que residen en hogares con jefatura femenina en términos de acceso a la salud, sino que establece diferencias en el acceso al mercado de trabajo formal, pues la carencia de obra social es un indicador utilizado por la Organización Internacional del Trabajo (OIT) para caracterizar la calidad del empleo e identificar la informalidad laboral (Vezza y Bertranou, 2011). Es muy probable que las jefas de hogar compatibilicen sus compromisos domésticos ingresando al mercado laboral en puestos flexibles y de fácil entrada y salida, características propias de la mayor parte de las ocupaciones del sector informal. 
Por otra parte, la pobreza monetaria y la percepción de una ayuda social también afectan con mayor intensidad a los hogares encabezados por mujeres y contribuyen fuertemente a la brecha de género. Si bien las transferencias de dinero o en especie son útiles para mitigar ciertas carencias -por lo que se podría interpretar que recibir subsidios es positivo-, la privación consiste en depender de ese recurso precario debido a la inestabilidad o insuficiencia en la generación de ingresos corrientes. Probablemente, los hogares con jefes mujeres están más afectados por la privación subsidio porque los organismos benefactores habitualmente eligen a las "madres" como beneficiarias ${ }^{8}$. Por ejemplo, en el programa de transferencias monetarias condicionadas más importante de la Argentina, la Asignación Universal por Hijo para Protección Social (AUH), la prestación se abona solo a uno de los padres, privilegiando a las madres. Tal vez la implementación masiva de este programa sea la principal causa del aumento de 4,3 puntos porcentuales en el aporte de esta privación a la diferencia de género.

Por otra parte, el 10\% de la diferencia de género en 2015 se explica por el bajo nivel de educación de los jefes de hogar. En la Argentina, el nivel educativo medio de las mujeres es más alto que el de los hombres (11,8 en comparación con 11,3 años de estudio, respectivamente, en 2014). Sin embargo, se puede afirmar que entre la población multidimensionalmente pobre, las jefas de hogar están menos educadas que sus pares varones, lo que puede obedecer a situaciones que se remontan a etapas previas del curso de vida de las mujeres. Aun así, esta privación ha perdido participación en la diferencia de género total en los últimos 10 años.

En conjunto, las condiciones habitacionales explican alrededor de la cuarta parte de la brecha de género. La privación más relevante en 2015 era vivir en una zona de basural. Su participación aumentó casi 3 puntos porcentuales en los últimos 10 años. Le siguen, en orden de importancia, la falta de agua corriente por cañería dentro de la vivienda, el baño sin desagüe, las condiciones de hacinamiento y la carencia de cuarto de cocina. Mientras la falta de agua corriente es la única de esas privaciones cuya contribución también aumentó, el resto evidencia considerables reducciones y participaciones menores al $5 \%$.

El aporte de la privación "niños y adolescentes que no asisten a un establecimiento educativo" es relevante y registra un aumento en el período analizado. La mayor presencia de menores de 18 años en hogares con jefatura femenina es posiblemente la razón por la cual esta privación adquiere relevancia. Si bien las otras privaciones que integran la dimensión “inclusión económica y social" (personas que no estudian ni trabajan, desocupación y adultos mayores sin cobertura previsional) aumentan levemente, no registran una participación importante en la brecha total.

En suma, los hogares con jefatura femenina presentan condiciones más desfavorables que los hogares encabezados por hombres. Pese a que la diferencia en la pobreza multidimensional entre géneros ha disminuido en los últimos 10 años, su estructura permanece casi intacta y se observa el predominio de idénticas privaciones.

\footnotetext{
Esto tiene un sustento empírico importante si el objetivo de esos programas es modificar el consumo de las niñas y los niños que residen en el hogar. Como mostraron Case y Deaton (2002) con datos de la India y Sudáfrica, la composición del gasto del hogar es extremamente sensible al género del jefe del hogar.
} 


\section{Análisis condicional}

El cuadro 4 contiene los promedios de las variables que reflejan las características, también llamadas "atributos" o "dotaciones", del conjunto de hogares y de los hogares definidos por el sexo del jefe, en cada uno de los dos años analizados.

Cuadro 4

\section{Argentina: estadísticas descriptivas de variables relacionadas} con las privaciones múltiples, 2005 y 2015

\begin{tabular}{|c|c|c|c|c|c|c|}
\hline \multirow[b]{2}{*}{ Variables } & \multicolumn{3}{|c|}{2005} & \multicolumn{3}{|c|}{2015} \\
\hline & Todos & $\begin{array}{c}\text { Jefe } \\
\text { hombre }\end{array}$ & $\begin{array}{l}\text { Jefe } \\
\text { mujer }\end{array}$ & Todos & $\begin{array}{c}\text { Jefe } \\
\text { hombre }\end{array}$ & $\begin{array}{l}\text { Jefe } \\
\text { mujer }\end{array}$ \\
\hline Incidencia de la pobreza $(\mathrm{k}=3)$ & 0,359 & 0,342 & 0,411 & 0,251 & 0,232 & 0,289 \\
\hline \multicolumn{7}{|l|}{ Sexo del jefe del hogar } \\
\hline Mujer (referencia) & 0,243 & & & 0,335 & & \\
\hline Hombre & 0,757 & & & 0,665 & & \\
\hline \multicolumn{7}{|l|}{ Región de residencia } \\
\hline Gran Buenos Aires (referencia) & 0,538 & 0,552 & 0,494 & 0,515 & 0,526 & 0,494 \\
\hline Noroeste argentino & 0,096 & 0,087 & 0,124 & 0,098 & 0,091 & 0,114 \\
\hline Nordeste argentino & 0,051 & 0,047 & 0,062 & 0,052 & 0,050 & 0,057 \\
\hline Cuyo & 0,064 & 0,065 & 0,060 & 0,067 & 0,072 & 0,059 \\
\hline Centro & 0,228 & 0,225 & 0,237 & 0,230 & 0,226 & 0,237 \\
\hline Sur & 0,025 & 0,025 & 0,023 & 0,037 & 0,036 & 0,039 \\
\hline \multicolumn{7}{|l|}{ Edad del jefe del hogar } \\
\hline Menor de 25 años & 0,030 & 0,026 & 0,041 & 0,032 & 0,031 & 0,034 \\
\hline 25-59 años (referencia) & 0,750 & 0,781 & 0,652 & 0,724 & 0,753 & 0,669 \\
\hline Mayor de 59 años & 0,220 & 0,192 & 0,307 & 0,244 & 0,217 & 0,297 \\
\hline $\begin{array}{l}\text { Educación del jefe del hogar } \\
\text { (en años de estudio) }\end{array}$ & 8,9 & 9,1 & 8,4 & 9,5 & 9,5 & 9,3 \\
\hline \multicolumn{7}{|l|}{ Situación laboral del jefe del hogar } \\
\hline Asalariado formal (referencia) & 0,315 & 0,361 & 0,172 & 0,383 & 0,443 & 0,265 \\
\hline Asalariado informal & 0,208 & 0,189 & 0,270 & 0,149 & 0,141 & 0,166 \\
\hline Independiente & 0,168 & 0,194 & 0,085 & 0,142 & 0,173 & 0,081 \\
\hline Patrón & 0,052 & 0,061 & 0,023 & 0,045 & 0,061 & 0,013 \\
\hline Desocupado & 0,056 & 0,056 & 0,057 & 0,023 & 0,019 & 0,030 \\
\hline Inactivo & 0,201 & 0,139 & 0,393 & 0,258 & 0,164 & 0,444 \\
\hline Ocupados en el hogar & 1,6 & 1,7 & 1,4 & 1,6 & 1,7 & 1,5 \\
\hline \multicolumn{7}{|l|}{ Estado civil del jefe del hogar } \\
\hline Casado (referencia) & 0,751 & 0,918 & 0,231 & 0,712 & 0,886 & 0,366 \\
\hline Separado & 0,088 & 0,026 & 0,279 & 0,104 & 0,033 & 0,243 \\
\hline Viudo & 0,093 & 0,024 & 0,307 & 0,090 & 0,028 & 0,212 \\
\hline Soltero & 0,068 & 0,031 & 0,182 & 0,095 & 0,052 & 0,180 \\
\hline \multicolumn{7}{|l|}{ Miembros en el hogar } \\
\hline Menores de 18 años & 1,6 & 1,7 & 1,5 & 1,5 & 1,5 & 1,5 \\
\hline Mayores de 70 años & 0,1 & 0,0 & 0,1 & 0,0 & 0,0 & 0,0 \\
\hline
\end{tabular}

Fuente: Elaboración propia, sobre la base de datos del Instituto Nacional de Estadística y Censos (INDEC), Encuesta Permanente de Hogares (EPH)

Nota: Datos correspondientes al segundo trimestre de 2005 y 2015. 
Como se analizó en la sección anterior, la proporción de personas con privaciones múltiples, medida en este caso por el indicador de incidencia $(\mathrm{H})$ con un umbral de tres privaciones, se redujo 10,7 puntos porcentuales entre 2005 y 2015 y es claramente mayor en los hogares con jefatura femenina en los dos años analizados. Además, hubo un aumento de casi 9 puntos porcentuales en la proporción de personas que residen en hogares con jefatura femenina.

Una generalidad que se destaca es la ventaja de los hogares con jefatura masculina en ciertas variables clave: promedio de años de escolaridad, proporción de jefes asalariados no registrados, desocupados e inactivos. Estos resultados permiten predecir que los niveles de pobreza en los hogares con jefatura femenina serán mayores que en los hogares con jefatura masculina, que es lo que efectivamente se observa.

Todas estas diferencias entre uno y otro tipo de hogares fueron disminuyendo con el paso del tiempo, probablemente debido a mejoras en aquellos con jefatura femenina y no a un empeoramiento de la situación de los hogares con jefatura masculina. Por ejemplo, los años de escolaridad aumentaron más en los hogares con jefatura femenina, mientras que la reducción del porcentaje de jefas de hogar asalariadas informales fue el doble de la de jefes asalariados informales.

Dado que no puede saberse a priori qué parte de la brecha se debe a que se están comparando universos diferentes, se estimó un modelo multivariante con el doble propósito de comparar grupos homogéneos y descomponer la brecha en la parte debida a dotaciones o características y la parte que queda sin explicar por esos factores y que se adjudican aquí a los parámetros de las regresiones. En el cuadro 5 se resumen los resultados obtenidos. Las columnas 1 a 3 contienen los correspondientes al año 2005, mientras que las columnas 4 a 5 los relativos a 2015. Los modelos explican en todos los casos más del 30\% de la varianza del indicador de pobreza utilizado $(\mathrm{H})$. No obstante lo anterior, más que el poder explicativo global del modelo interesa ver la significación de los parámetros individuales estimados (para cada variable).

En general, se observa que la mayor parte de las variables está fuertemente correlacionada con la probabilidad de que una persona resida en un hogar privado multidimensionalmente y que los signos encontrados corresponden a las previsiones ${ }^{9}$. Queda claro que, al menos en el año 2005, no se aprecian diferencias de incidencia en múltiples privaciones entre hogares con jefatura de diferente género (obsérvese la significación de la variable "jefe hombre"), y que la significación no es muy alta en 2015 (significativa al 5\%), de manera que la brecha de privaciones entre hogares con jefatura masculina y hogares con jefatura femenina pasa de 5,7 (encontrados a nivel descriptivo) a 1,5 puntos porcentuales ${ }^{10}$. Esto significa que las disparidades encontradas y analizadas en el examen descriptivo previo se deben en gran medida (si no en su totalidad, como en 2005) a las diferentes dotaciones de los jefes de hogar, que por lo general son más bajas en las jefas mujeres.

9 Si bien la unidad de observación es el hogar, las regresiones se estimaron con respecto a las personas. Por definición, una persona es pobre si reside en un hogar identificado como pobre.

10 Es más, si se cambia el modelo a un logit, por ejemplo, la significatividad estadística desaparece, con lo cual no se puede afirmar de manera contundente que la diferencia entre hogares con jefatura masculina y femenina exista. 
Cuadro 5

\section{Argentina: resultados de la regresión probit acerca de la probabilidad de una persona de residir en un hogar con al menos tres privaciones, 2005 y 2015}

\begin{tabular}{|c|c|c|c|c|c|c|}
\hline & \multicolumn{3}{|c|}{2005} & \multicolumn{3}{|c|}{2015} \\
\hline & Todos & Hombres & Mujeres & Todos & Hombres & Mujeres \\
\hline & 1 & 2 & 3 & 4 & 5 & 6 \\
\hline \multirow[t]{2}{*}{ Jefe hombre } & $-0,008$ & & & $-0,015^{\star \star}$ & & \\
\hline & $(0,012)$ & & & $(0,007)$ & & \\
\hline \multicolumn{7}{|l|}{ Región de residencia } \\
\hline \multirow[t]{2}{*}{ Noroeste argentino } & $0,094^{\star * *}$ & $0,100^{* * *}$ & $0,070^{* * *}$ & 0,001 & 0,011 & $-0,019^{*}$ \\
\hline & $(0,009)$ & $(0,010)$ & $(0,019)$ & $(0,006)$ & $(0,007)$ & $(0,011)$ \\
\hline \multirow[t]{2}{*}{ Nordeste argentino } & $0,059^{* \star *}$ & $0,052^{* * *}$ & $0,071^{\star * *}$ & $0,015^{\star *}$ & $0,017^{\star \star}$ & 0,011 \\
\hline & $(0,010)$ & $(0,012)$ & $(0,022)$ & $(0,007)$ & $(0,008)$ & $(0,013)$ \\
\hline \multirow[t]{2}{*}{ Cuyo } & $-0,076^{\star \star \star}$ & $-0,060^{\star \star \star}$ & $-0,140^{\star \star *}$ & $-0,093^{\star \star \star}$ & $-0,093^{\star \star \star}$ & $-0,085^{\star * \star}$ \\
\hline & $(0,009)$ & $(0,011)$ & $(0,020)$ & $(0,005)$ & $(0,005)$ & $(0,012)$ \\
\hline \multirow[t]{2}{*}{ Centro } & $-0,058^{\star \star \star}$ & $-0,048^{\star * *}$ & $-0,091^{\star * *}$ & $-0,056^{\star \star \star}$ & $-0,047^{\star \star \star}$ & $-0,076^{\star * \star}$ \\
\hline & $(0,008)$ & $(0,008)$ & $(0,017)$ & $(0,005)$ & $(0,006)$ & $(0,011)$ \\
\hline Patagonia & $-0,146^{\star \star \star}$ & $-0,158^{\star \star *}$ & $-0,103^{\star \star \star}$ & $-0,101^{\star \star \star}$ & $-0,095^{\star \star \star}$ & $-0,115^{\star * \star}$ \\
\hline Edad del jefe del hogar & $(0,010)$ & $(0,011)$ & $(0,026)$ & $(0,005)$ & $(0,006)$ & $(0,011)$ \\
\hline \multirow[t]{2}{*}{ Menor de 25 años } & $0,213^{* * *}$ & $0,268^{* \star *}$ & 0,067 & $0,231^{* \star *}$ & $0,220^{\star \star *}$ & $0,233^{\star * *}$ \\
\hline & $(0,022)$ & $(0,025)$ & $(0,042)$ & $(0,025)$ & $(0,032)$ & $(0,038)$ \\
\hline \multirow[t]{2}{*}{ Mayor de 59 años } & $-0,047^{\star \star *}$ & $-0,006$ & $-0,116^{\star \star \star}$ & $-0,076^{\star \star *}$ & $-0,069^{\star \star \star}$ & $-0,086^{* * *}$ \\
\hline & $(0,011)$ & $(0,013)$ & $(0,021)$ & $(0,007)$ & $(0,008)$ & $(0,015)$ \\
\hline \multirow[t]{2}{*}{ Educación } & $-0,067^{\star \star \star}$ & $-0,066^{\star \star *}$ & $-0,070^{\star * *}$ & $-0,047^{\star * *}$ & $-0,044^{\star \star \star}$ & $-0,053^{* * *}$ \\
\hline & $(0,001)$ & $(0,001)$ & $(0,003)$ & $(0,001)$ & $(0,001)$ & $(0,002)$ \\
\hline \multicolumn{7}{|c|}{ Situación laboral del jefe del hogar } \\
\hline \multirow[t]{2}{*}{ Informal } & $0,361^{\star \star \star}$ & $0,352^{\star \star \star}$ & $0,424^{\star \star \star}$ & $0,313^{\star \star *}$ & $0,332^{\star \star \star}$ & $0,246^{\star * *}$ \\
\hline & $(0,011)$ & $(0,013)$ & $(0,026)$ & $(0,011)$ & $(0,013)$ & $(0,021)$ \\
\hline \multirow[t]{2}{*}{ Independiente } & $0,272^{\star \star *}$ & $0,258^{* \star *}$ & $0,328^{\star * *}$ & $0,237^{\star \star \star}$ & $0,247^{\star \star \star}$ & $0,147^{\star \star \star}$ \\
\hline & $(0,012)$ & $(0,012)$ & $(0,033)$ & $(0,011)$ & $(0,012)$ & $(0,024)$ \\
\hline \multirow[t]{2}{*}{ Patrón } & $-0,060^{\star \star \star}$ & $-0,053^{\star *}$ & $-0,141^{\star \star *}$ & $0,081^{\star * *}$ & $0,096^{\star \star \star}$ & $-0,023$ \\
\hline & $(0,020)$ & $(0,021)$ & $(0,054)$ & $(0,016)$ & $(0,016)$ & $(0,057)$ \\
\hline \multirow[t]{2}{*}{ Desocupado } & $0,630^{\star \star \star}$ & $0,623^{\star \star \star}$ & $0,636^{\star * *}$ & $0,531^{* * *}$ & $0,566^{\star \star \star}$ & $0,452^{\star \star \star}$ \\
\hline & $(0,010)$ & $(0,012)$ & $(0,013)$ & $(0,028)$ & $(0,037)$ & $(0,045)$ \\
\hline \multirow[t]{2}{*}{ Inactivo } & $0,257^{* \star \star}$ & $0,179^{\star \star \star}$ & $0,407^{\star * *}$ & $0,172^{\star \star *}$ & $0,163^{\star \star \star}$ & $0,148^{\star \star *}$ \\
\hline & $(0,014)$ & $(0,018)$ & $(0,027)$ & $(0,012)$ & $(0,016)$ & $(0,018)$ \\
\hline \multirow[t]{2}{*}{ Ocupados en el hogar } & 0,001 & $-0,005$ & $0,018^{\star \star *}$ & $0,011^{* * *}$ & 0,002 & $0,029^{\star * *}$ \\
\hline & $(0,004)$ & $(0,005)$ & $(0,007)$ & $(0,003)$ & $(0,003)$ & $(0,005)$ \\
\hline \multicolumn{7}{|l|}{ Estado civil del jefe } \\
\hline \multirow[t]{2}{*}{ Separado } & $0,052^{\star \star \star}$ & $0,053^{*}$ & $0,043^{*}$ & $0,027^{\star \star}$ & $0,056^{\star \star \star}$ & 0,023 \\
\hline & $(0,016)$ & $(0,029)$ & $(0,022)$ & $(0,011)$ & $(0,018)$ & $(0,015)$ \\
\hline \multirow[t]{2}{*}{ Viudo } & $-0,081^{\star \star \star}$ & $-0,046^{\star \star}$ & $-0,113^{\star * \star}$ & $0,018^{*}$ & 0,023 & 0,016 \\
\hline & $(0,013)$ & $(0,023)$ & $(0,023)$ & $(0,011)$ & $(0,019)$ & $(0,016)$ \\
\hline Soltero & $0,061^{* * *}$ & $0,084^{\star \star \star}$ & $0,053^{\star *}$ & $0,080^{\star \star *}$ & $0,066^{\star * *}$ & $0,101^{\star \star *}$ \\
\hline & $(0,017)$ & $(0,025)$ & $(0,026)$ & $(0,012)$ & $(0,018)$ & $(0,016)$ \\
\hline Miembros en el hogar & & & & & & \\
\hline Menores de 18 años & $0,125^{\star \star \star}$ & $0,120^{\star \star \star}$ & $0,139^{\star \star \star}$ & $0,094^{\star \star \star}$ & $0,083^{\star \star \star}$ & $0,116^{\star \star *}$ \\
\hline & $(0,003)$ & $(0,003)$ & $(0,005)$ & $(0,002)$ & $(0,002)$ & $(0,004)$ \\
\hline Mayores de 69 años & $-0,022$ & $-0,005$ & $-0,059^{\star *}$ & $0,037^{\star \star \star}$ & $0,052^{\star \star \star}$ & $-0,007$ \\
\hline & $(0,014)$ & $(0,016)$ & $(0,027)$ & $(0,013)$ & $(0,016)$ & $(0,019)$ \\
\hline Observaciones & 46843 & 34515 & 12328 & 59967 & 39109 & 20858 \\
\hline Pseudo $\mathrm{R}^{2}$ & 0,364 & 0,357 & 0,394 & 0,344 & 0,347 & 0,341 \\
\hline
\end{tabular}

Fuente: Elaboración propia, sobre la base de datos del Instituto Nacional de Estadística y Censos (INDEC), Encuesta Permanente de Hogares (EPH).

Nota: Datos correspondientes al segundo trimestre de 2015. Los parámetros estimados son significativos al: ${ }^{* * *} 1 \%,{ }^{*} 5 \%,{ }^{*} 10 \%$. Se incluye entre paréntesis el error estándar robusto. 
Los datos del cuadro 5 permiten constatar que, sin diferenciar por el sexo del jefe del hogar, la probabilidad de que una persona resida en un hogar con privaciones múltiples decrece con la edad y con el nivel educativo del jefe del hogar y aumenta con el número de niños y de adultos mayores. También puede apreciarse que las personas residentes en un hogar encabezado por un jefe inactivo, desempleado o con un empleo informal, tienen una probabilidad mayor que el resto, de sufrir privaciones múltiples. Un hallazgo curioso corresponde a la variable "ocupados en el hogar". Nótese que el signo positivo indicaría que un mayor número de ocupados en el hogar acrecienta la probabilidad de que sus miembros sufran privaciones. Una explicación posible de este resultado es precisamente la causalidad de la relación. Más que causalidad, el modelo capta relaciones entre variables, con lo cual resulta plausible que en un hogar con privaciones aumente la necesidad de que sus miembros ingresen al mercado de trabajo, con respecto a un hogar con menos privaciones.

Muchos parámetros estimados en las regresiones cambiaron a lo largo de la década analizada. Entre ellos se destacan los relativos a la educación del jefe del hogar, a su inserción laboral y al estado civil. Pero lo que reviste mayor interés para el presente artículo tiene que ver con las diferencias en los parámetros estimados entre hogares con jefatura masculina y hogares con jefatura femenina.

Ciertas variables influyen con intensidad similar en la probabilidad de experimentar privaciones, independientemente del sexo del jefe del hogar. Tal vez las diferencias más importantes correspondan al trabajo, tanto remunerado como no remunerado. Puede verse que las variables relacionadas con el trabajo remunerado inciden con mayor intensidad en los hogares con jefatura masculina, mientras que el cuidado de niños (trabajo no remunerado) incide de manera más acentuada en los hogares con jefatura femenina. Este hallazgo relativo al mercado de trabajo solo vale para 2015, pues en 2005 la probabilidad de que los miembros del hogar sufrieran privaciones múltiples era mayor en los hogares encabezados por mujeres.

Lo más interesante en este caso tiene que ver justamente con los cambios ocurridos entre 2005 y 2015 en los hogares con jefatura femenina. Nótese que la probabilidad de experimentar privaciones en los hogares regidos por una mujer que trabaja de manera informal, está desempleada o es inactiva ha disminuido ostensiblemente. A su vez, pierde significancia el estado civil, que resultaba central en 2005 , y se reduce también la probabilidad de sufrir privaciones en alguna dimensión según el número de niños en el hogar. Esto refleja un progreso notable en el período de referencia, que disminuyó la propensión a la privación por los factores mencionados. 


\section{Microdescomposición de las diferencias}

En este apartado se analizan los resultados obtenidos tras aplicar un procedimiento de microdescomposición, que consiste en estimar el peso de lo que podrían llamarse "dotaciones" o "características" de los hogares (mostradas en el cuadro 4) y separarlo del peso de lo que podrían denominarse "propensiones" o "parámetros" (mostradas en el cuadro 5) y que reflejan la manera en que uno y otro tipo de hogar "transforman" esas dotaciones o características en mayor o menor probabilidad de experimentar privaciones. Los resultados se presentan en el cuadro 6 .

Cuadro 6

Argentina: diferencia condicional y no condicional del total de privaciones y de privaciones por dimensión, 2005 y 2015

\begin{tabular}{|c|c|c|c|c|c|}
\hline \multirow{3}{*}{ Dimensiones } & \multicolumn{2}{|c|}{ Brecha } & \multicolumn{3}{|c|}{ Fuente de la diferencia } \\
\hline & Condicional & $\begin{array}{c}\text { No } \\
\text { condicional }\end{array}$ & Dotaciones & Parámetros & Interacción \\
\hline & 1 & 2 & 3 & 4 & 5 \\
\hline \multicolumn{6}{|l|}{ Panel A (2005) } \\
\hline \multirow[t]{2}{*}{ Todas } & $-0,008$ & $0,068^{* * *}$ & $0,064^{\star \star \star}$ & 0,016 & $-0,011$ \\
\hline & $(0,012)$ & $(0,007)$ & $(0,009)$ & $(0,010)$ & $(0,012)$ \\
\hline \multirow[t]{2}{*}{ 1. Capacidad económica } & $-0,031^{\star \star \star}$ & $0,075^{\star \star \star}$ & $0,042^{\star \star *}$ & 0,003 & $0,036^{\star \star \star}$ \\
\hline & $(0,013)$ & $(0,007)$ & $(0,009)$ & $(0,010)$ & $(0,012)$ \\
\hline \multirow[t]{2}{*}{ 2. Vivienda } & $-0,033^{* \star *}$ & $0,039^{* * *}$ & 0,030 & $0,049^{* \star *}$ & $-0,040^{\star \star \star}$ \\
\hline & $(0,009)$ & $(0,006)$ & $(0,008)$ & $(0,010)$ & $(0,012)$ \\
\hline \multirow[t]{2}{*}{ 3. Saneamiento } & $-0,017^{* * *}$ & $0,018^{\star *}$ & $0,035^{\star * \star}$ & $0,068^{* * *}$ & $-0,086^{\star \star \star}$ \\
\hline & $(0,009)$ & $(0,006)$ & $(0,008)$ & $(0,011)$ & $(0,012)$ \\
\hline \multirow[t]{2}{*}{ 4. Inclusión } & 0,017 & $-0,021^{* * *}$ & $-0,010$ & $-0,023^{\star *}$ & 0,012 \\
\hline & $(0,011)$ & $(0,007)$ & $(0,009)$ & $(0,012)$ & $(0,013)$ \\
\hline \multirow[t]{2}{*}{ 5. Salud } & $-0,040^{* * *}$ & $0,116^{\star * \star}$ & $0,119^{\star \star \star}$ & $0,063^{* * *}$ & $-0,065^{\star \star \star}$ \\
\hline & $(0,013)$ & $(0,007)$ & $(0,009)$ & $(0,011)$ & $(0,012)$ \\
\hline \multicolumn{6}{|l|}{ Panel B (2015) } \\
\hline \multirow[t]{2}{*}{ Todas } & $-0,015^{\star \star}$ & $0,056^{\star \star \star}$ & $0,050^{\star \star \star}$ & $0,027^{\star \star \star}$ & $-0,020^{\star \star \star}$ \\
\hline & $(0,007)$ & $(0,005)$ & $(0,006)$ & $(0,007)$ & $(0,007)$ \\
\hline \multirow[t]{2}{*}{ 1. Capacidad económica } & $-0,024^{\star \star \star}$ & $0,082^{\star \star \star}$ & $0,054^{\star \star \star}$ & 0,008 & $0,019^{\star \star}$ \\
\hline & $(0,009)$ & $(0,006)$ & $(0,006)$ & $(0,008)$ & $(0,008)$ \\
\hline \multirow[t]{2}{*}{ 2. Vivienda } & $-0,029^{\star \star \star}$ & $0,027^{\star \star \star}$ & 0,001 & $0,043^{\star \star \star}$ & $-0,018^{\star \star}$ \\
\hline & $(0,007)$ & $(0,005)$ & $(0,005)$ & $(0,008)$ & $(0,008)$ \\
\hline \multirow[t]{2}{*}{ 3. Saneamiento } & $-0,009^{*}$ & $0,010^{\star *}$ & 0,007 & $0,026^{\star * \star}$ & $-0,023^{\star * \star}$ \\
\hline & $(0,005)$ & $(0,005)$ & $(0,005)$ & $(0,007)$ & $(0,007)$ \\
\hline \multirow[t]{2}{*}{ 4. Inclusión } & $0,020^{\star \star \star}$ & $-0,005$ & $-0,011^{\star}$ & $-0,007$ & 0,009 \\
\hline & $(0,004)$ & $(0,005)$ & $(0,006)$ & $(0,008)$ & $(0,008)$ \\
\hline \multirow[t]{2}{*}{ 5. Salud } & $-0,036^{\star * *}$ & $0,089^{\star * *}$ & $0,078^{* * *}$ & $0,033^{* * *}$ & $-0,022^{\star \star \star}$ \\
\hline & $(0,009)$ & $(0,006)$ & $(0,007)$ & $(0,008)$ & $(0,008)$ \\
\hline
\end{tabular}

Fuente: Elaboración propia, sobre la base del Instituto Nacional de Estadística y Censos (INDEC), Encuesta Permanente de Hogares (EPH)

Nota: Datos correspondientes al segundo trimestre de 2016. Los parámetros estimados son significativos al ${ }^{\star * *} 1 \%,{ }^{* \star} 5 \%,{ }^{*} 10 \%$. Se incluye entre paréntesis el error estándar robusto. 
En la columna 1 se muestra la brecha ajustada (o condicionada), es decir la que resulta de la variable "jefe hombre" incluida en los modelos multivariantes. El panel A corresponde a 2005 y el panel B a 2015. En la columna 2 figura la brecha "bruta" o no ajustada, que surge directamente de los datos. Las columnas 3 a 5 contienen la descomposición de la brecha "bruta" en la parte debida a dotaciones (columna 3), a los $\beta$ 's (columna 4) y a la interacción entre dotaciones y parámetros. En este artículo interesa en particular la columna 3, que es la parte estrictamente "explicada" de la brecha. En el cuadro 6 se incluyen también los resultados del cómputo y la descomposición de las disparidades entre géneros en cada una de las dimensiones que se trabajaron. En las filas del cuadro se detalla la brecha en capacidad económica, vivienda, saneamiento, inclusión y salud, y se sigue con la misma estructura anterior (brecha condicional, no condicional y descomposición) para cada una de ellas.

Este ejercicio de descomposición muestra que el $94 \%$ de la brecha de privaciones de 2005 y el $89 \%$ de la de 2015 pueden explicarse por diferencias en lo que aquí se denominó "dotaciones". Esta es una enseñanza muy útil para las políticas públicas. Ya se vio antes que el nivel educativo de las jefas de hogar, su situación en el mercado de trabajo y sobre todo su estado civil pueden representar desventajas y generar riesgos de privaciones múltiples para ellas mismas y para los miembros de los hogares que sostienen.

Cabe preguntarse entonces en qué dimensiones es más intenso el "efecto dotación" que se ha verificado en todas las privaciones. Hay varias situaciones particulares a nivel de dimensiones. Excepto en el caso de la inclusión social, la importancia explicativa de las dotaciones disminuye. No sorprende que "inclusión" sea la dimensión más atípica, pues la disparidad es muy reducida y la diferencia de la brecha no ajustada no es significativa.

Esto implica que la reducción de la disparidad observada a nivel descriptivo (una brecha que pasó del 6,8\% al 5,6\%) se debió principalmente a una disminución de la brecha de dotaciones entre los géneros y la brecha que persiste es todavía explicada en buena medida por la disparidad de esas dotaciones. Al analizar cada dimensión por separado se descubre que el poder explicativo de las dotaciones es menor en casi todos los casos, excepto en la dimensión "inclusión", que presenta un comportamiento anómalo.

\section{Conclusiones}

En este trabajo se analizó la brecha de pobreza entre las personas que residen en hogares con jefatura masculina y aquellas que residen en hogares con jefatura femenina con el objeto de presentar resultados que ayuden a focalizar mejor las políticas públicas de alivio de la pobreza. Mediante un análisis multidimensional se procura identificar los factores generales y, en particular, de género que predisponen más o menos a la situación de pobreza.

Los resultados muestran una disminución de la pobreza multidimensional entre 2005 y 2015, coincidente con la recuperación económica que experimentó la Argentina después de la grave crisis de 2001-2002. En ese período se observa una reducción de la brecha entre los hogares regidos por jefes de diferente sexo. Lo curioso de esta evolución es que al condicionar los datos, la disparidad entre géneros desaparece en 2005 y sigue siendo positiva, significativa y favorable 
a los hogares con jefatura masculina en 2015. Condicionar significa, en este caso, comparar hogares y jefes de hogar idénticos en la medida en que lo permiten los datos disponibles. Desde esta perspectiva la brecha de género no solo no disminuyó sino que aumentó.

El análisis de descomposición permitió establecer que la mayor proporción de las diferencias en los niveles de pobreza según el sexo del jefe del hogar se debe a lo que en este artículo se denominó diferencias en "dotaciones". Esto significa que los hogares encabezados por mujeres no son más proclives a la pobreza por una actitud discriminatoria hacia ellas, sino porque las jefas de hogar tienen menor nivel educativo que los jefes varones, su inserción en el mercado de trabajo es más precaria y viven solas, por lo que tienen que soportar una carga demográfica sin ayuda de otros adultos. No obstante, hay un porcentaje menor que estaría explicado por la discriminación o el trato diferente de las mujeres.

A partir de los resultados obtenidos se puede inferir que las mejoras en la inserción laboral de los pobres en general, y de las mujeres en particular, tendrían importantes repercusiones en la reducción de la pobreza. Estas políticas deberían diseñarse teniendo en cuenta la necesidad de cuidado de familiares a cargo y la calidad del empleo, pues la vulnerabilidad entre las mujeres que viven solas y tienen hijos es alta, y gran parte de la brecha de género está explicada por la falta de obra social, que puede entenderse como un indicador de la precariedad de los trabajos realizados por las mujeres a lo largo de su ciclo de vida.

\section{Bibliografía}

Agbodji, A. E., Y. M. Batana y D. Ouedraogo (2013), "Gender inequality in multidimensional welfare deprivation in West Africa: the case of Burkina Faso and Togo", Policy Research Working Paper Series, $\mathrm{N}^{\circ}$ 6522, Washington, D.C., Banco Mundial, junio.

Alkire, S. y J. Foster (2011), "Counting and multidimensional poverty measurement", Journal of Public Economics, vol. $95, \mathrm{~N}^{\circ} 7-8$, Amsterdam, Elsevier, agosto.

_ (2007), "Recuento y medición multidimensional de la pobreza", OPHI Working Paper Series, $\mathrm{N}^{\circ} 7$, Oxford, Universidad de Oxford, diciembre.

Alkire, S. y M. E. Santos (2014), "Measuring acute poverty in the developing world: robustness and scope of the multidimensional poverty index", World Development, vol. 59, Amsterdam, Elsevier, julio.

_ (2010), "Acute multidimensional poverty: a new index for developing countries", OPHI Working Paper Series, $\mathrm{N}^{\circ}$ 38, Oxford, Universidad de Oxford, julio. Alkire, S. y otros (2015), Multidimensional Poverty Measurement and Analysis, Oxford, Oxford University Press.

Arévalo, C. y J. Paz (2015), "Pobreza en la Argentina. Privaciones múltiples y asimetrías regionales", Documentos de Trabajo, $\mathrm{N}^{\circ}$ 15, Salta, Instituto de Estudios Laborales y del Desarrollo Económico (IELDE).

Ariza, M. y O. de Oliveira (2001), "Familias en transición y marcos conceptuales en redefinición", Papeles de Población, vol. 7, $\mathrm{N}^{\circ}$ 28, Toluca, Universidad Autónoma del Estado de México, abril-julio.

Arriagada, I. (2005), "Dimensiones de la pobreza y políticas desde una perspectiva de género", Revista de la CEPAL, No 85 (LC/G.2266-P), Santiago, Comisión Económica para América Latina y el Caribe (CEPAL), abril.

Atkinson, A. (2003), "Multidimensional deprivation: contrasting social welfare and counting approaches", Journal of Economic Inequality, vol. 1, No 1, Dordrecht, Kluwer Academic Publishers, abril.

Barros, R.P., M. De Carvalho y S. Franco (2006), "Pobreza multidimensional no Brasil", Texto para discusión, $\mathrm{N}^{\circ} 1227$, Río de Janeiro, Instituto de Investigación Económica Aplicada (IPEA), octubre. 
Battiston, D. y otros (2009), "Income and beyond: multidimensional poverty in six Latin American countries", OPHI Working Paper Series, $\mathrm{N}^{\circ} 17$, Oxford, Universidad de Oxford, septiembre.

Beccaria, L. y A. Minujin (s/f), "Métodos alternativos para medir la evolución del tamaño de la pobreza", Documentos de Trabajo, № 6, Buenos Aires, Instituto Nacional de Estadística y Censos (INDEC).

Blinder, A. S. (1973), "Wage discrimination: reduced form and structural estimates", The Journal of Human Resources, vol. 8, $\mathrm{N}^{\circ}$ 4, Madison, University of Wisconsin Press.

Boltvinik, J. (1992), "El método de medición integrada de la pobreza. Una propuesta para su desarrollo", Comercio Exterior, vol. 42, $\mathrm{N}^{\circ}$ 4, Ciudad de México, Banco Nacional de Comercio Exterior, abril.

Bossert, W., S. Chakravarty y C. D'Ambrosio (2009), "Multidimensional poverty and material deprivation", ECINEQ Working PaperSeries, $\mathrm{N}^{\circ} 129$, Society for the Study of Economic Inequality (ECINEQ), septiembre.

Bourguignon, F. y S. Chakravarty (2003), "The measurement of multidimensional poverty", Journal of Economic Inequality, vol. 1, $\mathrm{N}^{\circ}$ 1, Dordrecht, Kluwer Academic Publishers, abril.

Case, A. y A. Deaton (2002), "Consumption, health, gender and poverty", Centerfor Health and Wellbeing Working Papers, $\mathrm{N}^{\mathrm{O}}$ 21, Princeton, Princeton University, julio.

Chakravarty, S. (1997), "On Shorrocks' reinvestigation of the Sen Poverty Index", Econometrica, vol. 65, $\mathrm{N}^{\circ} 5$, Nueva York, Econometric Society, septiembre.

Cid, J. (2013a), "La pobreza en la Argentina y las estrategias de los hogares", Reunión de Discusión, $\mathrm{N}^{\circ} 194$, Salta, Universidad Nacional de Salta, septiembre.

(2013b), "Las estrategias de subsistencia de los hogares argentinos", documento presentado en el Tercer Seminario Anual de Economía Regional, Salta, 10 de octubre.

(1994), ¿Son los pobres de Salta más intensamente pobres?, Salta, Dirección General de Estadísticas y Censos de Salta, inédito.

CEPAL (Comisión Económica para América Latina y el Caribe) (2013), Panorama Social de América Latina (LC/G.2580), Santiago.

(2010), Pobreza infantil en América Latina y el Caribe (LC/R.2168), Santiago.

Conconi, A. (2009), "Pobreza multidimensional en la Argentina: ampliando las medidas tradicionales de pobreza por ingresos y NBI", tesis de maestría, La Plata, Universidad Nacional de La Plata.

Conconi, A. y A. Ham (2007), "Pobreza relativa multidimensional: una aplicación a la Argentina", Documentos de Trabajo, $\mathrm{N}^{\circ}$ 57, La Plata, Centro de Estudios Distributivos Laborales y Sociales (CEDLAS), agosto. CONEVAL (Consejo Nacional de Evaluación de la Política de Desarrollo Social) (2009), Metodología para la medición multidimensional de la pobreza en México, Ciudad de México.

Cortés, F. (1997), "Determinantes de la pobreza de los hogares. México, 1992", Revista Mexicana de Sociología, vol. 59, $\mathrm{N}^{\circ}$ 2, Ciudad de México, Universidad Nacional Autónoma de México, abril-junio.

Chakravarty, S. R. y C. D'Ambrosio (2006), "The measurement of social exclusion", Review of Income and Wealth, vol. 52, $\mathrm{N}^{\circ} 3$, Oxford, Blackwell Publishing, septiembre.

Chakravarty, S., J. Deutsch y J. Silber (2008), "On the watts multidimensional poverty index and its decomposition", World Development, vol. 36, N 6, Amsterdam, Elsevier, junio.

Fairlie, R. (2005), "An extension of the Blinder-Oaxaca decomposition technique to logit and probit models", Journal of Economic and Social Measurement, vol. 30, $\mathrm{N}^{\circ}$ 4, IOS Press, noviembre.

Feres, J. y X. Mancero (2001), "El método de las necesidades básicas insatisfechas (NBI) y sus aplicaciones en América Latina" (LC/L.1491-P), serie Estudios Estadísticos y Prospectivos, $\mathrm{N}^{\circ} 7$, Santiago, Comisión Económica para América Latina (CEPAL), febrero.

Foster, J. (1998), "Absolute versus relative poverty", The American Economic Review, vol. 88, $\mathrm{N}^{\circ}$ 2, Nashville, American Economic Association, mayo.

Foster, J. y A. Sen (1997), "On economic inequality. After a quarter century", On Economic Inequality, Oxford, Oxford University Press.

Gordon, D. y otros (2003), Child Poverty in the Developing World, Bristol, The Policy Press, octubre.

Hicks, N. y P. Streeten (1979), "Indicators of development: the search for a basic needs Yardstick", World Development, vol. 7, N 6, Oxford, Pergamon Press Ltd., junio. 